\title{
Giardiasis y desnutrición infantil
}

\author{
Giardiasis and child malnutrition
}

Alejandra Zapata Morales ${ }^{\top}$; Luisa Fernanda Arboleda Restrepo ;

Luz Nelly Díaz Ramírez

Tutor: Aura María Gil Villa²

\section{Resumen}

Se revisaron 67 artículos sobre giardiasis y desnutrición infantil publicados en las bases de datos Scientific Electronic Library Online y Pubmed entre el año 1998 y 2015, para recopilar información relacionada con la prevalencia de la giardiasis y de la desnutrición infantil e identificar posibles estrategias de tratamiento y prevención. La giardiasis es una enfermedad intestinal parasitaria, generalmente asintomática, causada por el protozoo Giardia lamblia. Las manifestaciones clínicas se caracterizan por diarrea, dolor abdominal, pérdida de peso, síndrome de malabsorción y desnutrición, lo cual se explica por el comportamiento fisiopatológico del parásito sobre los enterocitos y la oclusión intestinal que impide la absorción de los nutrientes y, por tanto, el desarrollo en talla y peso del niño. En Colombia, la prevalencia de la Giardia es del $12 \%$ en la población general y del $28 \%$ entre niños de 1 y 4 años. En diversos estudios realizado en América, África y Asia se muestra una relación estadísticamente significativa entre los niños infectados con Giardia lamblia, la restricción del crecimiento, el bajo peso y/o la desnutrición leve a crónica. Se concluyó que las infecciones por parásitos intestinales tienen una elevada prevalencia y sus consecuencias se acentúan aún más en las personas de estrato socioeconómico bajo que viven en inadecuadas condiciones de vida como el hacinamiento, la mala disposición de las basuras, dificultades en el acceso de agua potable, los malos hábitos de higiene y desnutrición. Se ha observado que en los países en vía de desarrollo, se encuentra disminuido el control frente a los enteros parásitos debido a los costos 
de financiación, la falta de infraestructura para un adecuado diagnóstico y la escasez de proyectos educativos dirigidos a la comunidad.

Palabras clave: asintomático, infección, niños.

\section{Abstract}

A total of 67 papers on giardiasis and child malnutrition published in Scientific Electronic Library Online and PubMed databases between 1998 and 2015 were reviewed in order to collect information related to the prevalence of giardiasis and child malnutrition and identify possible treatment and prevention strategies. Giardiasis is an intestinal parasitic disease, generally asymptomatic, caused by the protozoan Giardia lamblia. Clinical manifestations include diarrhea, abdominal pain, weight loss, malabsorption syndrome and malnutrition, which is explained by the parasite pathophysiological behavior on enterocytes and intestinal obstruction which prevents the absorption of nutrients and the subsequent height and weight development. In Colombia, the prevalence of Giardia is reported to be $12 \%$ among the general population and $28 \%$ among children aged 1 to 4 years. Several studies conducted in America, Africa and Asia found a statistically significant relationship between children infected with Giardia lamblia, decreased growth, low-weight-for-age and/or mild to chronic malnutrition. This paper concludes that intestinal parasitic infections show a high prevalence and their consequences are much greater among people with low socioeconomic status living under inadequate conditions including overcrowding, poor garbage disposal, limited access to drinking water, unhygienic practices and malnutrition. It has been observed that in developing countries there is reduced control of enteric-parasites due to financial costs, the lack of infrastructure for a proper diagnosis and the scarcity of educational projects for the community.

Keywords: asymptomatic, infection, children.

\section{Introducción}

Millones de niños enfrentan la vida con el hambre y la malnutrición dedibo a la desigualdad social en los países, entendida como un malestar ligado a la economía y a las clases sociales (Save the Children Everyone, 2008). Además, deben enfrentarse con las enfermedades que son consecuencia de su estado de desnutrición como la giardiasis, el parásito intestinal más común entre los niños, con mayor prevalencia en aquellos que asisten a la guardería o están inmunosuprimidos y hace que su estado de desnutrición empeore y su tratamiento sea más complejo (Heresi, Murphy, y Cleary, 2000).

La giardiasis es una enfermedad causada por un parásito protozoario flagelado, extracelular y anaerobio; binucleado, de simetría bilateral y que se reproduce por fusión binaria, llamado Giardia lamblia también conocido como $G$. intestinalis y como G. duodenalis (Hawrelak, 2003). La Giardia tiene la capacidad de adoptar dos formas: trofozoito móvil y quiste infectante. El quiste tiene forma ovalada, posee cuatro núcleos y doble membrana, lo cual lo protege de la cloración, las temperaturas extremas, la deshidratación y le permite sobrevivir en aguas frías durante varias semanas (Weller, 2012).

Durante su ciclo de vida, la forma infectante se ingiere generalmente a través del agua o de alimentos contaminados con quistes o por contacto oro-fecal. Una vez ingerido el parásito, llega al estómago donde se reblandece y después en el duodeno, el trofozoito se adhiere al epitelio intestinal del huésped, a través de la cara ventral que posee un disco similar a una ventosa. Lo anterior, origina el borramiento de las vellosidades intestinales, el cual es el mecanismo principal fisiopatológico del 
parásito, que ocasiona malabsorción de nutrientes y disminución de las enzimas digestivas. Posteriormente, el parásito se enquista de nuevo y es excretado al medio externo, completando así el ciclo de vida (Escobedo, 2014), (Alparo, 2005) (Figura 1).

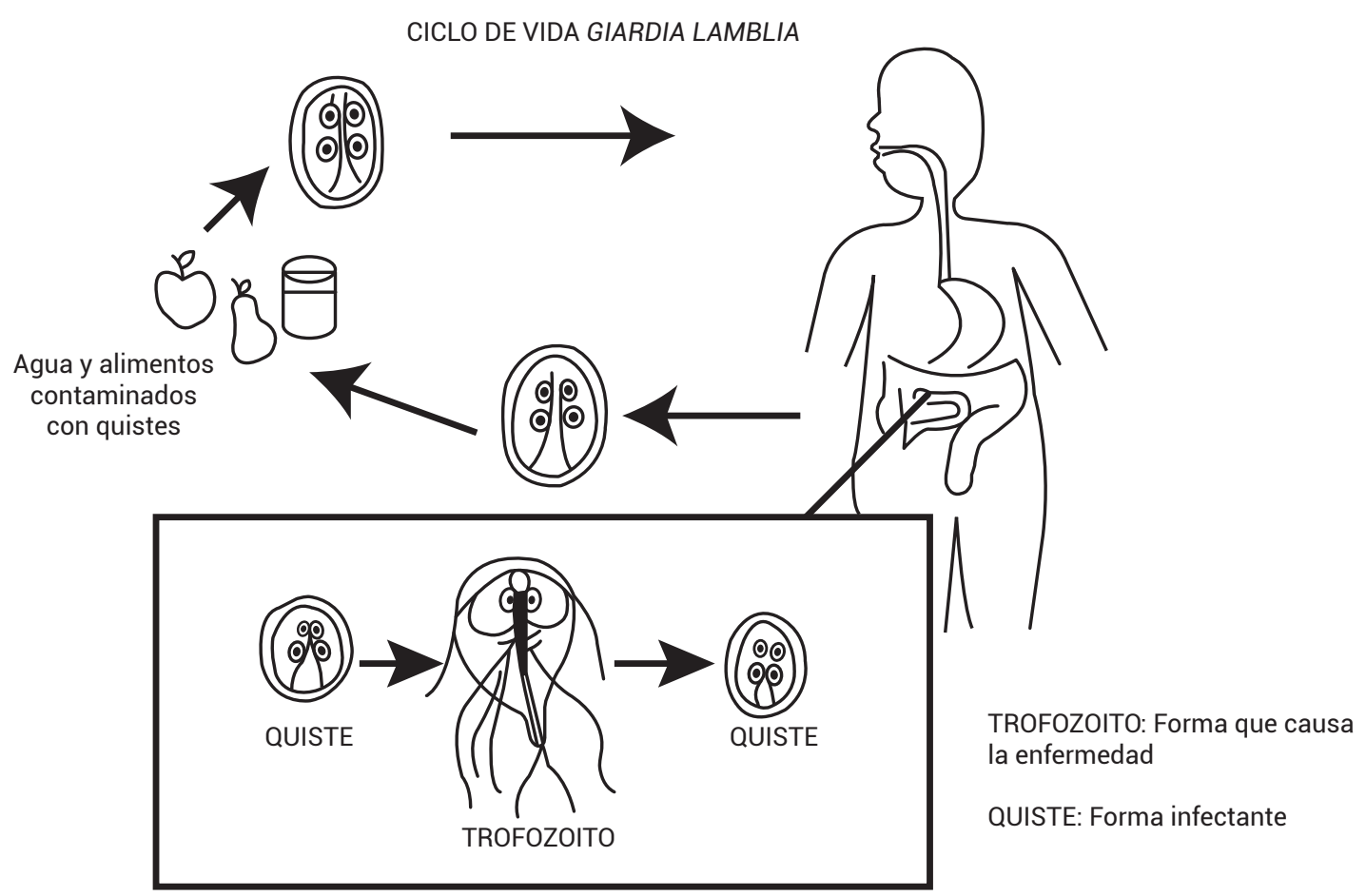

Figura 1. Ciclo de vida de la Giardia lamblia.

Fuente. Modificado de Center for Disease Control and Prevention, CDC 2012.

\section{Epidemiología}

La Giardia lamblia es el protozoo que con mayor frecuencia se encuentra en pruebas copro-parasitológicas, estimándose que alrededor de 280 millones de personas en todo el mundo presentan giardiasis (Tsuji, 2009; Botero, Garcés, García Montoya, Grisales Patiño, Aguirre Acevedo, y Álvarez Uribe, 2009; Carvalho-Costa, 2007). En los países industrializados, la prevalencia de Giardia lamblia, oscila entre el 2 y el 5\%; en Europa Occidental y Estados Unidos la infección por este parásito se asocia con su ingestión en aguas contaminadas, transmisión de persona a persona y viaje a zonas endémicas (Prado, 2005; Almirall, 2013).

Los países en vías de desarrollo, tropicales y subtropicales son más propensos a presentar infecciones parasitarias; en África, Asia y Latinoamérica, la giardiasis es endémica y cerca de 200 millones de personas están infectadas. Los niños contraen giardiasis a temprana edad con una prevalencia del 15 al $20 \%$ en niños menores de 10 años, y alcanza su pico de incidencia en niños de 2 a 12 años de edad, particularmente en la población desnutrida (Cabrera et al., 2005; Hartree, 2010). 
En Colombia, la prevalencia de Giardia lamblia es del $12 \%$ en la población general y del $28 \%$ entre niños de 1 y 4 años (Luna, Camacho, Rojas, y Bayona, 2010). En el año 2006 se realizó un estudio de prevalencia de Giardia lamblia en la región antioqueña, encontrándose que el $27.6 \%$ de los niños menores de 5 años estaban infectados con el parásito y, entre ellos, el $8.1 \%$ tenían bajo peso debido a un deficiente estado nutricional; sin embargo, son pocos los estudios que evalúan las frecuencias de esta enfermedad en nuestro país y específicamente en Antioquia donde persisten poblaciones con una alta susceptibilidad de sufrir infecciones por el microorganismo en cuestión (Botero-Garcés, García-Montoya, Grisales-Patiño, AguirreAcevedo, y Álvarez-Uribe, 2009; Londoño, Á. M., 2009) (Tabla 1).

Tabla 1. Prevalencia de Giardia lamblia en niños entre los 6 meses y los 15 años, entre el año de 1999 al 2014 en diferentes departamentos de Colombia.

\begin{tabular}{|c|c|c|}
\hline \multicolumn{3}{|c|}{ DEPARTAMENTO DE ANTIOQUIA } \\
\hline AUTORES & PREVALENCIA DE GIARDIA LAMBLIA & AÑo \\
\hline (Botero Garcés et al., 2009) & $\begin{array}{l}\text { El } 27.3 \% \text { de los niños desde los } 8 \text { meses hasta } 6 \\
\text { años estaban infectados con guardia. }\end{array}$ & Octubre 2006 \\
\hline (Carmona, 2009) & $\begin{array}{l}\text { Prevalencia de } 21.2 \% \text { en Turbo y el Bagre en niños } \\
\text { malarios entre los } 4 \text { a los } 10 \text { años }\end{array}$ & $\begin{array}{l}\text { Julio del } 2004 \text { a julio } \\
\text { del } 2005\end{array}$ \\
\hline (Tabares, 2008) & $\begin{array}{l}\text { Prevalecía de } 24.7 \% \text { en niños menores de } 12 \text { años } \\
\text { en la vereda La Doctora. }\end{array}$ & $\begin{array}{l}\text { Junio2005 y mayo } \\
2007\end{array}$ \\
\hline \multicolumn{3}{|c|}{ DEPARTAMENTO DE ATLÁNTICO } \\
\hline (Londoño J. C., 2005) & $\begin{array}{l}\text { Prevalencia de } 36.1 \% \text { en niños entre los } 2 \text { a } 6 \text { años } \\
\text { asistentes a hogares del ICBF. }\end{array}$ & $\begin{array}{l}\text { Entre enero y marzo } \\
2004\end{array}$ \\
\hline \multicolumn{3}{|c|}{ DEPARTAMENTO DE BOYACÁ } \\
\hline (Manrique Abril y Suescún Carrero, 2011) & $\begin{array}{l}\text { Prevalencia del } 12.4 \% \text { en menores de } 5 \text { años con } \\
\text { EDA en consulta externa en el hospital San Rafael } \\
\text { y en la Clínica Saludcoop de Tunja. }\end{array}$ & Durante el año 2004 \\
\hline \multicolumn{3}{|c|}{ DEPARTAMENTO DE CUNDINAMARCA } \\
\hline (Chaves, 2007) & $\begin{array}{l}\text { Prevalencia en niños de } 1 \text { a } 5 \text { años. } \\
\text { 13.60\% en el año } 1995 . \\
12.84 \% \text { en el año } 2001 . \\
15.16 \% \text { en el año } 2005 .\end{array}$ & Año 2005 \\
\hline \multicolumn{3}{|c|}{ DEPARTAMENTO DE QUINDÍO } \\
\hline (Lora Suárez et al., 2002) & $\begin{array}{l}\text { Prevalencia de } 60.4 \% \text { en niños entre los } 3 \text { y } 13 \\
\text { años. }\end{array}$ & En el año 1999 \\
\hline $\begin{array}{l}\text { (Giraldo Gómez, Lora, Henao, Mejía y Gómez } \\
\text { Marín, 2005) }\end{array}$ & $\begin{array}{l}\text { Prevalencia del } 13 \% \text { en niños con edades preesco- } \\
\text { lares de madres comunitarias del ICBF. }\end{array}$ & Año 2003 al 2004 \\
\hline (Londoño, Mejía y Gómez Marín, 2009) & $\begin{array}{l}\text { Prevalencia de } 13.2 \% \text { en niños entre los } 6 \text { y } 60 \text { me- } \\
\text { ses de estrato } 1 \text { y } 2 \text {, asistentes a hogares infanti- } \\
\text { les en el área de Calarcá. }\end{array}$ & Año 2004-2005 \\
\hline (Arias, Guzmán, Lora, Torres y Gómez, 2010) & $\begin{array}{l}\text { Prevalencia de } 16 \% \text { en niños de } 2 \text { a } 5 \text { años asis- } \\
\text { tentes a hogares infantiles en Circasia. }\end{array}$ & Año 2008 \\
\hline \multicolumn{3}{|c|}{ DEPARTAMENTO DEL TOLIMA } \\
\hline (Rodríguez et al., 2014) & Prevalencia de $11.17 \%$ en niños en el ICBF. & Año 2009 \\
\hline
\end{tabular}

Fuente. Elaboración propia. 


\section{Diagnóstico}

Existen diferentes métodos diagnósticos para la detección de la Giardia lamblia como el examen coprológico, el drenaje del contenido duodenal y pruebas inmunológicas que se basan en la detección de antígeno-anticuerpo. La eficiencia en la detección de la giardia en heces ha sido del $76 \%$, y dependiendo de las réplicas puede llegar hasta el $85 \%$. La sensibilidad diagnóstica del análisis citológico del contenido duodenal puede alcanzar el $96 \%$, pero es un método altamente invasivo (Vásquez Zubieta, 1995). Por su parte, las pruebas inmunológicas se consideran un importante método que ha logrado alcanzar sensibilidades del $99 \%$ y, además, representan una buena alternativa costo-beneficio (Cacciò, 2004). La prueba de ELISA es un análisis inmunoabsorbente ligado a enzimas, el cual consiste en detectar la reacción antígeno-anticuerpo mediante la utilización de enzimas que ayudan a acelerar la reacción; tiene una alta sensibilidad aproximada de 95100\% (Medical Chemical Corporation, 2010).

\section{Manifestaciones clínicas}

La giardiasis puede causar diversas manifestaciones clínicas desde un síndrome diarreico agudo, crónico o intermitente, hasta un síndrome de malabsorción de nutrientes. Las alteraciones del tracto gastrointestinal producen dolor abdominal intenso, náuseas, y vómitos; la diarrea se presenta con heces de características lientéricas, pastosas y malolientes, y los síntomas varían según las características del huésped (Quesada, 2002).

Aproximadamente, del 60 al $80 \%$ de los casos de giardiasis permanecen como portadores asintomáticos, en su gran mayoría son niños. De hecho, los niños menores de 5 años, poseen mayores factores de riego de desarro-
Ilar la infección por su conducta para la edad: continencia fecal disminuida, frecuente contacto persona-persona y exploración por la boca, con la consiguiente succión digital y de objetos. El problema principal de la población infantil menor de 5 años radica en que esta población es susceptible de presentar cuadros de malnutrición severa por la falta de tratamiento oportuno a la giardiasis (Gardner, 2001).

\section{Tratamiento de la Giardiasis}

La giardiasis puede ser auto limitada en la mayoría de los casos y principalmente en los adultos; sin embargo, debido a la aparición potencial de síntomas intermitentes o crónicos en los niños, se sugiere que debe ser tratada siempre y a la mayor brevedad posible (Yereli, 2004). Los nitroimidazoles son el tipo de medicamentos más utilizados para el tratamiento contra la infección por Giardia lamblia; como tratamiento primario son empleados el tinidazol en dosis única, metronidazol o benzimidazol de 5 a 7 días (Nash, 2001; Redondo Granado, 2009; Almirall et al., 2013). En algunos países el tratamiento primario se complementa a través de una combinación de dieta alimenticia y/o fitoterapia con el ajo, el propóleo y la pimienta larga de la india, lo cual ayuda a inhibir el desarrollo, la replicación y la adhesión de la Giardia a los enterocitos intestinales, (Hawrelak, 2003). De otro lado, se han probado nuevos fármacos in vitro contra la giardiasis, pero hasta ahora no se ha demostrado la eficacia en modelos animales o no hay una clara comprensión de los mecanismos de acción (Huamán-E, 2013).

\section{Prevención}

La Giardia lamblia está presente en ambientes urbanos, periurbanos y rurales en los que 
predominan higiene deficiente y hacinamiento. Algunas de las prácticas que se deben tener en cuenta para evitar la infección por Giardia son el lavado de manos, que es una práctica simple y eficaz para prevenir la infección; la utilización de agua hervida y/o filtrada en países en vía de desarrollo, donde el agua no es potable; la buena higiene en la manipulación de alimentos, y la utilización de métodos de barrera en las relaciones sexuales también es una forma de prevención de la infección (Gómez, 2003; De la Mata, 2008).

\section{Desnutrición infantil}

La desnutrición como definición global es un desequilibrio entre el escaso suministro de nutrientes y las altas demandas de crecimiento, mantenimiento y actividades específicas del cuerpo. Cuando un individuo no puede ingerir o asimilar los nutrientes suficientes para cubrir sus necesidades, el cuerpo empieza a consumir sus reservas conllevando a la pérdida de grasa, proteínas, vitaminas, minerales, hormonas, y de manera compensatoria en respuesta al catabolismo acelerado (Yereli, 2004).

El estado nutricional está determinado por factores ambientales, genéticos, neuroendocrinos y por la etapa biológica del desarrollo en el que se encuentre el individuo (Alcázar, Ocampo, Huamán, y Aparco, 2013). Por su parte, la desnutrición infantil se reconoce como uno de los principales problemas de salud pública y bienestar social de Latinoamérica, ya que es una de las mayores causas de morbimortalidad evitable en niños. La desnutrición infantil se encuentra relacionada con los bajos recursos socioeconómicos, situaciones precarias de hi- giene y de abandono de los niños, y con las políticas de salud de la gran mayoría de los países de Latinoamérica, lo cual empeora las formas graves de la desnutrición asociada con la infección por giardiasis (Alvear, L. y Kac, 2010; Gómez, 2003).

\section{Epidemiología}

La comparación de las relaciones entre el peso, la talla y la edad del individuo evaluado con un valor poblacional de referencia es la herramienta que permite establecer las diferentes clases de alteraciones nutricionales (Rivera, 2002). Para ello, es importante tener en cuenta los siguientes tres indicadores: (1) peso para la edad, para medir la desnutrición global, (2) talla para la edad, que determina la desnutrición crónica, debido a que la baja estatura es producto de una carencia prolongada de nutrientes, y (3) peso para la talla, que evalúa la desnutrición aguda (Ortiz-Andrellucchi, 2006; Damián, 2007).

En el año 2011, la desnutrición crónica en el mundo afectó a 531,000 niños entre cero y cinco años (19.5\% del total de niños de ese grupo de edad) (Rivera-Dommarco et al., 2013). Por su parte, en Colombia el $12 \%$ de los niños y de las niñas menores de cinco años presentaron desnutrición crónica, y el $7 \%$ presentaron desnutrición global; sin embargo, en la población infantil desplazada por la violencia, estos valores ascendieron al $23 \%$ y $14 \%$, respectivamente (Programa mundial de alimentos, 2010).

En la población infantil se describen dos grandes grupos clínicos de desnutrición: marasmo que es la pérdida excesiva de proteínas y energía, que lleva al organismo a un estado de caquexia, deteriorando el tejido adiposo y 
muscular (Uribe Gil, G y Alcaraz López, 2007) y el kwashiorkor, es la forma de desnutrición edematosa, caracterizada por la pérdida total de proteínas, y al igual que el marasmo puede presentarse a cualquier edad (Uribe Gil, 2007; Mejoramiento Alimentario y Nutricional de Antioquia, 2010).

\section{Tratamiento de la desnutrición}

Los niños con cuadros de desnutrición, ingresan al servicio de salud, principalmente con deshidratación como resultado de una diarrea de alto gasto, es decir, más de diez deposiciones liquidas por día, seguido de infecciones del tracto respiratorio, urinario o gastrointestinal. El tratamiento consiste en tres pilares fundamentales propuestos por la Gobernación de Antioquia. 1-Fase: crítica intrahospitalaria o estabilización clínica del paciente; 2-Fase: Recuperación nutricional; 3-Fase seguimiento médico, nutricional y psicosocial de manera ambulatoria (Mejoramiento Alimentario y Nutricional de Antioquia, 2010).

\section{Relación entre el estado nutricional infantil y la Giardiasis}

En el año 2008, Solano y sus colaboradores constataron un efecto deletéreo de las parasitosis sobre el estado nutricional de los niños (Solano, 2008). Por su parte, en dos estudios realizados en Colombia y chile se describe la asociación entre la desnutrición calórico-proteica en niños menores de cinco años y la infección por Giardia lamblia con consecuencias desfavorables sobre el peso y el crecimiento (Canales R., 2012). Sin embargo, en otro estudio realizado en niños menores de 5 años en la provincia de Buret, situada al sur de Ruanda, se encontró que la infección por Giardia duodenalis era menos frecuente en niños con déficit de hierro, comportándose como un factor protector (Danquah, 2014). En la Tabla 2 se observan diferentes estudios realizados desde el año 1999 hasta el año 2014, en América, Asia y África, en donde se describe una relación estadísticamente significativa entre los niños infectados con Giardia lamblia y la restricción del crecimiento, el bajo peso y/o la desnutrición leve a crónica.

Tabla 2. Relación entre la infección por Giardia lamblia y la desnutrición en niños desde el año 1999 al 2014.

\begin{tabular}{|l|l|c|}
\hline \multicolumn{1}{|c|}{ AUTORES } & \multicolumn{1}{|c|}{ RELACION DE GIARDIA LAMBLIA CON DESNUTRICIÓN } & \multicolumn{1}{|c|}{ AMÉRICA } \\
\hline (Silva, 2009) & $\begin{array}{l}\text { Prevalencia del 33.8\% de niños con giardiasis y desnutrición aguda. } \\
\text { Prevalencia de 29.1\% de niños con giardiasis y desnutrición crónica. }\end{array}$ & 2005 \\
\hline (Carvalho-Costa, 2007) & $\begin{array}{l}\text { Relación estadísticamente significativa entre desnutrición e infección con } \\
\text { Giardia lamblia. }\end{array}$ & 2005 \\
\hline (Botero Garcés et al., 2009) & $\begin{array}{l}\text { El 55.6\% de los infectados con Giardia lamblia presentaron desnutrición } \\
\text { crónica leve. }\end{array}$ & 2010 \\
\hline (Verhagen, 2013) & $\begin{array}{l}\text { Asociación estadísticamente significativa entre retardo en el crecimiento e } \\
\text { infección con Giardia lamblia. }\end{array}$ & 2010 \\
\hline (DuPont, 2013) & $\begin{array}{l}\text { Los niños con bajo peso por desnutrición preexistente, sufren de infección } \\
\text { persistente con Giardia lamblia con diarrea crónica. }\end{array}$ & 2013 \\
\hline (Bartelt, 2013) & $\begin{array}{l}\text { La infección persistente con Giardia lamblia se asocia a retardo en el } \\
\text { crecimiento. }\end{array}$ & 2013 \\
\hline (Vera Orellana, 2014) & $\begin{array}{l}\text { Existe mayor prevalencia de infección por Giardia lamblia en niños con re- } \\
\text { traso en el crecimiento. }\end{array}$ & 2014 \\
\hline
\end{tabular}




\begin{tabular}{|c|c|c|}
\hline AUTORES & RELACION DE GIARDIA LAMBLIA CON DESNUTRICIÓN & AÑo \\
\hline \multicolumn{3}{|c|}{ ASIA } \\
\hline (Mondal D. H., 2009) & $\begin{array}{l}\text { La restricción del crecimiento de los niños se asocia significativamente } \\
(95 \% \mathrm{Cl} 0.01-0.35) \text { con la diarrea asociada a E. histolítica, pero no a la } \\
\text { enfermedad diarreica asociada a Criptosporidio, Escherichia coli entero- } \\
\text { toxigénico, y Giardia. }\end{array}$ & 1999-2002 \\
\hline (Al-Mekhlafi M. S., 2005) & $\begin{array}{l}\text { La prevalencia de desnutrición fue estadísticamente significativa en infec- } \\
\text { tados con Giardia lamblia. }\end{array}$ & 2003 \\
\hline (Al-Mekhlafi M. H., 2007) & $\begin{array}{l}\text { La giardiasis se asoció significativamente con la baja concentración de } \\
\text { retinol en suero con un p valor de } 0.038 \text {. }\end{array}$ & 2004 \\
\hline (Al-Mekhlafi M. S., 2005) & Giardia lamblia fue el principal predictor de retraso en el crecimiento. & 2005 \\
\hline (Mondal D. M., 2012) & $\begin{array}{l}\text { Infectados por Giardia presentaron } 28.6 \%-34.0 \% \text { de bajo peso y } 16.3 \% \text { - } \\
42.4 \% \text { de retardo en el crecimiento. }\end{array}$ & 2008 \\
\hline (Al-Mekhlafı H. M. M., 2013) & Prevalencia de bajo peso de $28.3 \%$ en niños de 7 a 12 años con giardiasis. & 2013 \\
\hline \multicolumn{3}{|c|}{ ÁFRICA } \\
\hline (Nguyen, 2012) & $\begin{array}{l}\text { Los niños con peso inferior al normal tienen mayor probabilidad de con- } \\
\text { traer infección. }\end{array}$ & 2008 \\
\hline
\end{tabular}

Fuente. Elaboración propia.

\section{Conclusión}

Las infecciones por parásitos intestinales tienen una elevada prevalencia y sus consecuencias se acentúan aún más en las personas de estrato socioeconómico bajo que viven en inadecuadas condiciones de vida como el hacinamiento, la mala disposición de las basuras, dificultades en el acceso de agua potable, los malos hábitos de higiene y desnutrición. Se ha observado que las personas que residen en el área rural o en áreas en desarrollo son más propensas a infecciones parasitarias comparadas con los que habitan en el área urbana (Cabrera et al., 2005). Dentro de las infecciones por parásitos intestinales, Giardia lamblia es uno de los parásitos responsables de grandes problemas de salud pública en la mayoría de los países en desarrollo, en los cuales el control frente a los entero parásitos es difícil por los costos de financiación, la falta de infraestructura para un adecuado diagnóstico y la escasez de proyectos educativos dirigidos a la comunidad (Nkrumah, 2011).

\section{Referencias}

- Alcázar, L., Ocampo, D., Huamán, L., y Aparco, J. (2013). Impacto económico de la desnutrición cronica, aguda y global en el Perú. Revista Peruana de Medicina Experimental y Salud Publica, 30(4), 569-574.

Al-Mekhlafi, H. M., Al-Maktari, M. T., Jani, R., Ahmed, A., Anuar, T. S., Moktar, N., Surin, J. (2013). Burden of Giardia duodenalis Infection and Its Adverse Effects on Growth of Schoolchildren in Rural Malaysia. PLoS Neglected Tropical Diseases, 7(10), 1-12. doi:10.1371/journal.pntd.0002516

- Al-Mekhlafi, M. H., Azlin, M., Aini, U. N., Shaik, a., Sa'iah, a., y Norhayati, M. (2007). Prevalence and predictors of low serum retinol and hypoalbuminaemia among children in rural Peninsular Malaysia. Transactions of the Royal Society of Tropical Medicine and 
Hygiene, 101(12), 1233-1240. doi:10.1016/j. trstmh.2007.09.006

Al-Mekhlafi, M. S. H., Azlin, M., Nor Aini, U., Shaik, A., Sa'iah, A., Fatmah, M. S., Norhayati, M., et al. (2005a). Giardiasis as a predictor of childhood malnutrition in Orang Asli children in Malaysia. Transactions of the Royal Society of Tropical Medicine and Hygiene, 99, 686-691. doi:10.1016/j.trstmh.2005.02.006

Al-Mekhlafi, M. S. H., Azlin, M., Nor Aini, U., Shaik, A., Sa'iah, A., Fatmah, M. S., Norhayati, M. et al. (2005b). Giardiasis as a predictor of childhood malnutrition in Orang Asli children in Malaysia. Transactions of the Royal Society of Tropical Medicine and Hygiene, 99(9), 686-691. doi:10.1016/j.trstmh.2005.02.006

Almirall, P., Alfonso, M., Ávila, I., Salazar, Y., Escobedo, A. A., Núñez, F. A., y Cimerman, S. (2013). Clinical features of giardiasis in different age groups of pediatric in-patients. Revista Chilena de Infectología: Órgano Oficial de La Sociedad Chilena de Infectología, 30(5), 502-506. doi:10.4067/ s0716-10182013000500006

- Alparo Herrera, I. (2005). Giardiasis y desnutrición. Revista de La Sociedad Boliviana de Pediatría, 44(3), 166-173.

Alvear, G. G., L, J., y Kac. (2010). Epidemiología de la desnutrición en Latinoamérica: situación actual. Nutrición Hospitalaria, 25(3), 50-56.

Arias, J., Guzmán, G., Lora, F., Torres, E., y Gómez, J. (2010). Prevalencia de protozoos intestinales en 79 niños de 2 a 5 años de edad de un hogar infantil estatal en Circasia, Quindío. Revista Infectología, 14(1), 31-38. doi:10.1016/S0123-9392(10)70090-4
Bartelt, L. a, Roche, J., Kolling, G., Bolick, D., Noronha, F., Naylor, C., Guerrant, R. et al. (2013). Persistent G. lamblia impairs growth in a murine malnutrition model. The Journal of Clinical Investigation, 123(6). doi: 10.1172/JCI67294DS1

Botero, D y Restrepo, M. (1998). Parasitosis humanas. Medellín, Colombia: Corporación para Investigaciones Biológicas.

Botero-Garcés J. H., Garcia-Montoya G. M, Grisales-Patiño D., Aguirre-Acevedo D. C, Alvarez Uribe M. C. (2009). Giardia intestinalis and nutritional status in children participating in the complementary nutrition program, Antioquia, Colombia, May to October 2006. Rev Inst Med Trop Sao Paulo, 51(3), 155-162.

- Cabrera M, Verástegui, M., Cabrera, R. (2005). Prevalencia de enteroparasitosis en una comunidad altoandina de la Provincia de Víctor Fajardo, Ayacucho, Perú. Rev. Gastroenterol, 25 (2),150-155.

- Cacciò, S. M. (2004). New methods for the diagnosis of Cryptosporidium and Giardia. Parassitologia, 46(1-2),151-155. Recuperado de http://www.ncbi.nlm.nih. gov/pubmed/15305706

- Canales R. P., y Alliende G., F. (2012). Diarrea crónica en el niño. Revista Chilena de Pediatría, 83(2), 179-184. doi:10.4067/ S0370-41062012000200010

- Carmona Fonseca, J., Peñuela, R. M. U., y Botero, A. M. C. (2009). Parasitosis intestinal en niños de zonas palúdicas de Antioquia (colombia). Latreia, 22(1), 27-46.

Carvalho-Costa, F. A., Gonçalves, A. Q., Lassance, S. L., Silva Neto, L. M. da, Salmazo, 
C. A. A., y Bóia, M. N. (2007). Giardia lamblia and other intestinal parasitic infections and their relationships with nutritional status in children in Brazilian Amazon. Revista Do Instituto de Medicina Tropical de São Paulo, 49(3), 147-153. doi:10.1590/S003646652007000300003

- Chaves, P., Fernández, J. A., Ospina, I., López, M. C., Moncada, L., y Reyes, P. (2007). Tendencia de la prevalencia y factores asociados a la infección por Giardia duodenalis en escolares y preescolares de una zona rural de Cundinamarca. Biomedica, (27), 345-351.

- Damián, H. (2007). Praxis profesional y realidad clínica: la construcción de la desnutrición infantil como objeto terapéutico en un centro de atención primaria en la Ciudad de Buenos Aires. Cuadernos de Antropología Social, (25), 189-207.

Danquah, I., Gahutu, J. B., Ignatius, R., Musemakweri, A., y Mockenhaupt, F. P. (2014). Reduced prevalence of Giardia duodenalis in iron-deficient Rwandan children. Tropical Medicine and International Health, 19(5), 563-567. doi:10.1111/tmi.12284

- DuPont, H. L. (2013). Giardia: Both a harmless commensal and a devastating pathogen. Journal of Clinical Investigation, 123(6), 2352-2354. doi:10.1172/JCl69932

Escobedo, A. a., Almirall, P., Alfonso, M., Cimerman, S., y Chacín-Bonilla, L. (2014). Sexual transmission of giardiasis: A neglected route of spread? Acta Tropica, 132(1), 106-111. doi:10.1016/j.actatropica.2013.12.025

Field V. F., ford L, hill D. (2010). Giardiasis. National Travel Health Network and Centre.
Gardner, T. B., y Hill, D. R. (2001). Treatment of giardiasis. Clinical Microbiology Reviews, 14(1), 114-128. doi:10.1128/CMR.14.1.114128.2001

- Giraldo Gómez, J. M., Lora, F., Henao, L. H., Mejía, S., y Gómez Marín, J. E. (2005). Prevalencia de giardiasis y parásitos intestinales en preescolares de hogares atendidos en un programa estatal en Armenia, Colombia. Revista de Salud Pública, 7(3), 327,338. doi:10.1590/S012400642005000300008

- Gómez, F. (2003). Desnutrición. Salud Pública de México, 45(4), 576-582.

- Hawrelak, J. (2003). Giardiasis: Pathophysiology and Management. Journal of Clinical Therapeutic, 8(2), 129-142.

- Heresi, G.P., Murphy, J.R., Cleary, T.G. (2000). Giardiasis. Seminars in Pediatric Infectious Diseases, 11(3), 189-195. doi:10.1053/ pi. 2000.6230

- Kac G., García Alvear J. L. (2010). Epidemiología de la desnutrición en Latinoamérica: situación actual. Nutr. Hosp, 25(3), 50-56. Recuperado de http://scielo. isciii.es/scielo.php?script=sci_arttext\&pi$d=S 0212-16112010000900008 \&$ lng $=e s$

Londoño, A. L., Mejía, S., y Gómez Marín, J. E. (2009). Prevalence and risk factors associated with intestinal parasitism in preschool children from the urban area of Calarcá, Colombia. Revista de Salud Publica, 11(1), 72-81.

- Londoño, J. C., Hernández, A. P., Vergara, C., y Mareño, R. M. (2005). Parasitismo intestinal en hogares comunitarios. munici- 
pio de Santo Tomás. Colombia, Atlántico. Dugandia, 1(1), 59-66.

Lora Suárez, F., Marín Vásquez, C., Loango, N., Gallego, M., Torres, E., González, M. M.Gómez Marín, J. E. (2002). Giardiasis in children living in post-earthquake camps from Armenia (Colombia). BMC Public Health. doi:10.1186/1471-2458-2-5

Luna, D. M., Camacho, L., Rojas, D., y Bayona, M. A. (2010). Enteroparasitism prevalence in pre-scholar nurseries located near the low basis of the Tunjuelito river. Rev. U.D.C.A Act. \& Div. Cient. 13(1), 7-15. Recuperado de http://www.scielo. org.co/scielo.php?script=sci_arttext\&pi$d=S 0123-42262010000100002 \&$ Ing=en\&n$\mathrm{rm}=\mathrm{iso \&}$ tlng $=\mathrm{es}$

- Manrique-Abril, F. G., Billon Y Tigne, D., Bello, S. E., y Ospina, J. M. (2006). Agentes causantes de diarrea en niños menores de 5 años en Tunja, Colombia. Rev. salud pública, 8(1), 88-97. doi:10.1590/S012400642006000100008

- Mata C. de la. (2008). Malnutrición, desnutrición y sobrealimentación. Rev Med Rosario, 74, 17-20.

- Medical Chemical Corporation. (2010). Giardia lamblia Antigen Detection Microwell ELISA Directions For Use For In Vitro Diagnostic Use Catalog \# MCC-GA-96, 96 Test, 1-5. Recuperado de http://www. med-chem.com/pages/lab_procedures/ pdf/MCC\%20Giardia\%20ELISA\%20MCCGA-96\%2009-06-08.pdf

- Mejoramiento Alimentario y Nutricional de Antioquia. (2010). Manual para la atencion integral de los niños y niñas con desnutri- cion. MANA (Mejoramiento Alimentario y Nutricional de Antioquia).

Mondal, D., Haque, R., Sack, R. B., Kirkpatrick, B. D., y Petri, W. A. (2009). Short report: Attribution of malnutrition to cause-specific diarrheal illness: Evidence from a prospective study of preschool children in Mirpur, Dhaka, Bangladesh. American Journal of Tropical Medicine and Hygiene, 80(5), 824826. doi:80/5/824 [pii]

Mondal, D., Minak, J., Alam, M., Liu, Y., Dai, J., Korpe, P., Petri, W. A. et al. (2012). Contribution of enteric infection, altered intestinal barrier function, and maternal malnutrition to infant malnutrition in Bangladesh. Clinical Infectious Diseases, 54(2), 185-192. doi:10.1093/cid/cir807

Murray, Rosenthal y Pfaller. (2005). Medical Microbiology. Philadelphia, PA: Elsevier.

Nash, T.E.,Ohl, C. A., Thomas, E., Subramanian, G., Keiser, P., y Moore, Treatment of Patients with Refractory Giardiasis. Clinical Infectious Diseases, 33(1), 22-28. doi: 10.1086/320886

- Nguyen, N. L., Gelaye, B., Aboset, N., Kumie, A., Williams, M. A., y Berhane, Y. (2012). Intestinal parasitic infection and nutritional status among school children in Angolela, Ethiopia. Journal of Preventive Medicine and Hygiene, 53(3), 157-164.

- Nkrumah, B., y Nguah, S. B. (2011). Giardia lamblia: a major parasitic cause of childhood diarrhoea in patients attending a district hospital in Ghana. Parasites \& Vectors, 4(1), 163. doi:10.1186/1756-3305-4-163

Ortiz-Andrellucchi, A., Peña Quintana, L., Albino Beñacar, A., Mönckeberg Barros, F., y 
Serra-Majem, L. (2006). Desnutrición infantil, salud y pobreza: intervención desde un programa integral. Nutrición Hospitalaria, $21(4), 533-541$.

Prado, M. S., Cairncross, S., Strina, A., Barreto, M. L., Oliveira-Assis, A. M., y Rego, S. (2005). Asymptomatic giardiasis and growth in young children; a longitudinal study in Salvador, Brazil. Parasitology, 137(1), 51-56.

- Programa mundial de alimentos. (2010). Estado Nutricional, de Alimentación y Condiciones de Salud de la Población Desplazada por la Violencia en Seis Subregiones de Colombia | WFP | Programa Mundial de Alimentos - Luchando contra el hambre en el mundo. doi:10.1017/ s0031182005007353

- Quesada, M., Garita, M., y Fernández, A. (2002). Afecciones gastrointestinales vol III. Centro Nacional de Información de Medicamentos, 3, 1-88.

- Redondo Granado, M. J., y Díez Martín, M. C. (2009). Recaída de giardiasis tras el tratamiento con metronidazol. Pediatría Atención Primaria, 11(42), 333-335. doi:10.4321/ S1139-76322009000200013

- Rivera, M., de la Parte, M. A., Hurtado, P., Magaldi, L., y Collazo, M. (2002). Giardiasis Intestinal. Mini-Revisión. Investigación Clínica, 43(2), 119-128.

- Rivera-Dommarco, J. A., Cuevas-Nasu, L., González de Cosío, T., Shamah-Levy, T., y García-Feregrino, R. (2013). Desnutrición crónica en México en el último cuarto de siglo: análisis de cuatro encuestas nacionales. Salud Pública de México, 55, S161-S169.
Rodríguez, V., Espinosa, O., Carranza, J. C., Duque, S., Arévalo, A., Clavijo, J. A., Vallejo, G. A. et al. (2014). Contribución de los autores: Genotipos de Giardia duodenalis en muestras de niños de las guarderías del Instituto Colombiano de Bienestar Familiar y de perros en Ibagué, Colombia. Biomédica Tolima, 34, 271-281. doi:10.7705/biomedica.v34i2.1713

- Save the Children Everyone. (2008). A life free from hunger: tackling child malnutrition. Recuperado de http://www.savethechildren.org.uk/sites/default/files/docs/A-Life-Free-From-Hunger-UK-low-res.pdf

Silva, R. R., da Silva, C. a M., de Jesus Pereira, C. A., de Carvalho Nicolato, R. L., NegrãoCorrêa, D., Lamounier, J. A., y Carneiro, M. (2009). Association between nutritional status, environmental and socio-economic factors and Giardia lamblia infections among children aged 6-71 months in Brazil. Transactions of the Royal Society of Tropical Medicine and Hygiene, 103(5), 512519. doi:10.1016/j.trstmh.2008.10.019

Solano, L., Acuña, I., Barón, M. A., Moron de Salim, A., y Sánchez, A. (2008). Influencia de las parasitosis intestinales y otros antecedentes infecciosos sobre el estado nutricional antropométrico de niños en situación de pobreza. Parasitología Latinoamericana, 63(1-2-3-4), 12-19. doi:10.4067/S071777122008000100003

- Tabares, L. F. y Gómez, L. (2008). Prevalencia de parasitosis intestinales en niños menores de 12 años, hábitos higiénicos, características de las viviendas y presencia de bacterias en el agua en una vereda de Sabaneta, Antioquia, Colombia. Revista Médica Universidad de Antioquia. Latreia, 21(3), 253-259. 
- Tsuji, O. V., y Rivera, T. C. (2009). Giardiasis. La parasitosis más frecuente a nivel mundial. Revista del Centro de Investigación. Universidad La Salle, 8(31), 75-90.

- Uribe Gil, G. y Alcaraz López, G. (2007). El mal de ojo y su relación con el marasmo y kwashiorkor: El caso de las madres de Turbo, Antioquia, Colombia. Invest Educ Enferm, 25(2), 72-82.

- Vásquez Zubieta, F. (1995). Giardia lamblia: Estudio comparativo de tres métodos de diagnóstico, examen directo de heces, enterotest modificado y técnica de sedimentación espontánea en tubo. Acta médica sanmartiniana, 1(1), 33-37.

- Vera Orellana, M. de J., e Íñiguez Mendieta, R. C. (2014). Prevalencia y factores asociados de parasitismo escolar escolar en la escuela "Joaquín Fernández de Córdova" de 5 a 16 años. Periodo abril-septiembre 2014.
Recuperado de http://dspace.ucacue.edu. ec//handle/reducacue/5835

- Verhagen, L. M., Incani, R. N., Franco, C. R., Ugarte, A., Cadenas, Y., Sierra Ruiz, C. I. Pinelli, E. (2013). High Malnutrition Rate in Venezuelan Yanomami Compared to Warao Amerindians and Creoles: Significant Associations WITH Intestinal Parasites and Anemia. PLoS ONE, 8(10), 1-12. doi:10.1371/ journal.pone.0077581

Weller, Peter (2012). Infecciones por protozoarios y helmintos: generalidades. En Longo, Fauci, Kasper, Hauser, Jameson, Loscalzo. (Eds.), Principios de Medicina Interna (sección 17). México: Mc Graw Hill.

Yereli, K., Balciolu, I. C., Ertan, P., Limoncu, E., y Ona, A. (2004). Albendazole as an alternative therapeutic agent for childhood giardiasis in Turkey. Clinical Microbiology and Infection, 10(6), 527-529. 